\title{
Nutrition and health claims on healthy and less-healthy packaged food products in New Zealand
}

\author{
Haya H. Al-Ani ${ }^{1}$, Anandita Devi ${ }^{1}$, Helen Eyles ${ }^{1,2}$, Boyd Swinburn ${ }^{1,3}$ and Stefanie Vandevijvere ${ }^{1 *}$ \\ ${ }^{1}$ School of Population Health, The University of Auckland, Private Bag 92019, Auckland Mail Centre, Auckland 1142, \\ New Zealand \\ ${ }^{2}$ National Institute for Health Innovation, School of Population Health, The University of Auckland, Private Bag 92019, \\ Auckland Mail Centre, Auckland 1142, New Zealand \\ ${ }^{3}$ World Health Organization Collaborating Centre for Obesity Prevention, Deakin University, 221 Burwood Highway, \\ Melbourne, VIC 3125, Australia
}

(Submitted 2 February 2016 - Final revision received 1 July 2016 - Accepted 5 July 2016 - First published online 9 August 2016)

\section{Abstract}

Nutrition and health claims are displayed to influence consumers' food choices. This study assessed the extent and nature of nutrition and health claims on the front-of-pack of 'healthy' and 'less-healthy' packaged foods in New Zealand. Foods from eight categories, for which consumption may affect the risk of obesity and diet-related chronic diseases, were selected from the 2014 Nutritrack database. The internationally standardised International Network for Food and Obesity/Non-Communicable Diseases Research, Monitoring and Action Support (INFORMAS) taxonomy was used to classify claims on packages. The Nutrient Profiling Scoring Criterion (NPSC) was used to classify products as 'healthy' or 'less healthy'. In total, 7526 products were included, with $47 \%$ ( $n$ 3557) classified as 'healthy'. More than one-third of products displayed at least one nutrition claim and $15 \%$ featured at least one health claim on the front-of-pack. Claims were found on one-third of 'less-healthy' products; $26 \%$ of those products displayed nutrition claims and $7 \%$ featured health claims. About $45 \%$ of 'healthy' products displayed nutrition claims and $23 \%$ featured health claims. Out of 7058 individual claims, the majority (69\%) were found on 'healthy' products. Cereals displayed the greatest proportion of nutrition and health claims (1503 claims on 564 products), of which one-third were displayed on 'less-healthy' cereals. Such claims could be misleading consumers' perceptions of nutritional quality of foods. It needs to be explored how current regulations on nutrition and health claims in New Zealand could be further strengthened (e.g. using the NPSC for nutrition claims, including general health claims as per the INFORMAS taxonomy) to ensure consumers are protected and not misled.

Key words: Nutrition claims: Health claims: Food supply: New Zealand

More than 36 million people die annually from non-communicable diseases (NCD), comprising $63 \%$ of global deaths and including $>14$ million people who die prematurely between the ages of 30 and $70^{(1)}$ years. Obesity is an established risk factor for NCD, with high BMI as the world's leading modifiable risk to health ${ }^{(2,3)}$. The global prevalence of obesity has more than doubled since 1980 , with $11 \%$ of men and $15 \%$ of women aged 18 years and older estimated to be obese in $2014^{(3)}$. The most recent New Zealand Health Survey found that $31 \%$ of New Zealand adults and $10 \%$ of New Zealand children are obese. Moreover, obesity rates are disproportionately higher in Pacific (67\%) and Maori (46\%) adults compared with New Zealand European adults ${ }^{(4)}$.

A strong contributor to the significant burden of obesity and diet-related NCD is an unhealthy diet, fostered by an unhealthy food environment ${ }^{(5)}$. The 2013-2020 World Health Organization ${ }^{(1)}$ Global Action Plan provides an excellent overall guide for societal action for the prevention and control of NCD by creating health-promoting environments to reduce obesity, and underlying social determinants.

The International Network for Food and Obesity/NonCommunicable Diseases Research, Monitoring and Action Support (INFORMAS) is a global network of public-interest organisations and researchers that aims to monitor, benchmark and support public and private sector actions to create healthy food environments and reduce obesity, NCD and their related inequalities $^{(6)}$. INFORMAS recognises that nutrition labelling is an important component of a healthy food environment, with particular focus on front-of-pack (FoP) signposting and the

Abbreviations: FoP, front-of-pack; FSANZ, Food Standards Australia New Zealand; HFT, Heart Foundation Tick; INFORMAS, International Network for Food and Obesity/Non-Communicable Diseases Research, Monitoring and Action Support; NCD, non-communicable diseases; NIP, nutrition information panel; NPSC, Nutrient Profiling Scoring Criterion.

* Corresponding author: S. Vandevijvere, email s.vandevijvere@auckland.ac.nz 
regulation of nutrition and health claims ${ }^{(7)}$. INFORMAS has developed an internationally standardised taxonomy ${ }^{(7)}$ to classify nutrition and health claims on food products.

In New Zealand, nutrition claims ('any representation that states, suggests or implies that a food has particular nutritional properties including but not limited to the energy value and to the content of protein, fat and carbohydrates, as well as the content of vitamins and minerals') and health claims ('any representation that states, suggests or implies that a relationship exists between a food or a constituent of that food and health') ${ }^{(8)}$ are regulated by the Australia New Zealand Food Standards Code (FSC), and this regulation is implemented by the Ministry for Primary Industries $(9,10)$.

In accordance with the FSC, displaying a nutrition information panel (NIP) is mandatory in New Zealand on most packaged foods (displaying energy, protein, total fat, saturated fat, carbohydrate, sugars and $\mathrm{Na}$ per serving, and per $100 \mathrm{~g}$ or $100 \mathrm{ml}$ ), and if nutrition claims are featured the nutrition information for that nutrient must be displayed on the $\mathrm{NIP}^{(11)}$. However, the NIP is poorly understood by New Zealanders, particularly Maori, Pacific Islanders and low-income New Zealanders, who have disproportionately higher rates of obesity ${ }^{(12)}$. A new mandatory food standard (Standard 1.2.7) was implemented in January 2016 on the regulation of nutrition and health claims on food labels and in advertisements by Food Standards Australia New Zealand (FSANZ). This standard aims to reduce false and misleading nutrition claims and ensure that health claims are only present on foods meeting certain 'healthy' criteria ${ }^{(9)}$. A Nutrient Profiling Scoring Criterion (NPSC) was developed by FSANZ to determine the eligibility of foods to feature health claims ${ }^{(10)}$. The 'healthy' criteria set by the NPSC have been tested on $>10000$ New Zealand and Australian food products ${ }^{(13)} ; 59 \%$ of food products from seven food groups ( $n$ 550) met the 'healthy' criteria in New Zealand between 2007 and $2009^{(14)}$. Fewer than half (41\%) of all packaged foods available in New Zealand (41\%) and Australia (47\%) in 2012 met nutritional criteria to carry health claims ${ }^{(13)}$

Several industry and agency-initiated labelling systems operate in New Zealand. These include the Australian Food and Grocery Council's multi-icon Daily Intake Guide (DIG) system, individual logos and icons that relate to a particular issue (e.g. fair trade, organic), of which some are licence-based, such as the Heart Foundation Tick (HFT). Approximately 500 products currently display the DIG thumbnails in New Zealand; however, displaying percentage of dietary intake (DI) information is only mandatory for energy, whereas the use of additional percentage of DI information (fat, protein, saturated fat, carbohydrate, sugars and $\mathrm{Na}$ ) is voluntary ${ }^{(15)}$.

Given the significant influence of nutrition and health claims on consumers' food choices ${ }^{(16)}$, the aim of this study was to investigate the extent and nature of such claims on 'healthy' and 'less-healthy' packaged foods in New Zealand using the internationally standardised INFORMAS taxonomy ${ }^{(7)}$.

\section{Methods}

\section{Sampling}

Four of the biggest supermarkets in Auckland, New Zealand were chosen as sites for data collection (Countdown, PakNSave,
New World and 4Square). From these supermarkets, details of all food products available for purchase were recorded. Where the same product was sold in more than one supermarket, that product was included only once in the product sample.

\section{Data collection}

Nutritrack is a branded food and nutrient database, which contains annual package and nutrient information for all packaged foods on sale in four New Zealand supermarkets. From 20 February to 31 March 2014, photographs of the FoP, ingredient lists and NIP for each product were collected by trained fieldworkers using a specially developed smartphone application and are available in a web-based searchable database ${ }^{(17)}$. The brand name, product name, barcode and food composition information (per $100 \mathrm{~g} / \mathrm{ml}$ content of energy, protein, total fat, saturated fat, carbohydrates, sugar, fibre (only when present) and $\mathrm{Na}$ ) of the 2014 products selected for this study were exported to an Excel spreadsheet (Microsoft Excel 2010).

From the seventeen food categories available on the 2014 Nutritrack database of packaged food products in New Zealand, eight (including specific sub-categories) were selected, which were considered the healthiest and unhealthiest food categories as per a comparison of different New Zealand nutrient profiling systems: bakery products (biscuits, bread, cakes, muffins, pastries), cereals (breakfast cereals, cereal bars), confectionery (chocolate, sweets, jelly, cough lollies, chewing gum), convenience foods (pizza, salads, ready meals), dairy products (cheese, cream, desserts, ice cream and edible ices, milk, yogurt, yogurt drinks), fruit and vegetables, non-alcoholic beverages (beverage mixes, cordials, electrolyte drinks, fruit and vegetable juices, soft drinks, waters) and snack foods (crisps, snacks) (7976 of 14418 total Nutritrack products). All products within each category were included.

\section{Classification of health-related claims on front-of-pack of food products}

The internationally standardised taxonomy, developed by INFORMAS $^{(7)}$ and based on Codex food labelling standards ${ }^{(8)}$, was used to classify different types of claims featuring on FoP of food packages. Claims were classified into three categories: nutrition claims, health claims and 'other' claims, and then further divided into sub-categories (Table 1, online Supplementary Appendix S1). The category of 'Other' claims, such as 'gluten-free' or 'organic', will not be further discussed in this manuscript. Claim content was coded as detailed in the online Supplementary Appendix S1. All visible claims, including brand names, product names and slogans, were included as claims.

The definition of FoP was difficult for some food packages, such as cans, where claims were not completely visible without looking at all sides of the label. Such claims were not considered FoP, and therefore were not included. Products with non-English labels ( $n$ 53), found across five categories, were included with no claims recorded.

To classify claims according to the taxonomy, definitions were established to ensure consistent categorisation and added to the INFORMAS protocol. For example, the INFORMAS taxo- 
Table 1. Selected examples of classification of claims on food packages by claim type and content as per the International Network for Food and Obesity/Non-Communicable Diseases Research, Monitoring and Action Support taxonomy ${ }^{(7)}$

\begin{tabular}{lll}
\hline Types of claims & Example & Claim content \\
\hline Health-related ingredient claim & 'Contains 25\% fruit' & Fruits/nuts/honey \\
& 'Goodness of milk' & Milk/cream \\
Nutrient content claim & 'Low fat' & Fats \\
& 'High in fibre' & Fibre \\
Nutrient comparative claim & 'Sweetened with stevia plant' & Reduced sugar \\
& 'Lite' & Reduced fat \\
General health claim & Low Gl symbol & Low Gl/energy density \\
& 'FDI World Dental Federation approved' & Oral health \\
Nutrient and other function claim & 'Ca for strong bones' & Nutrient + bone \\
& 'Fibre for focus' & Nutrient + brain \\
Reduction of disease risk claim & 'Ca to reduce risk of osteoporosis' & Osteoporosis \\
& Heart Foundation Tick & Cardiovascular health \\
Other claim & '100\% natural ingredients' & Other health related \\
& 'Organic', 'fair trade' & Environment \\
\hline
\end{tabular}

GI, glycaemic index; FDI, Fédération dentaire internationale.

nomy defines a health-related ingredient claim as a claim that 'states, suggests or implies that a food has particular nutritional properties by virtue of its content of an ingredient ${ }^{\text {(7) }}$. Therefore, 'contains wholegrain' was considered a health-related ingredient claim, because it was thought that such a claim implied that the product had particular nutritional properties. However, a claim that a product contained fruit was not classified as a health-related ingredient claim unless the amount of that ingredient was specified; for example, 'contains blueberries' was not recorded as a claim, whereas 'contains five fruits' was classified as a health-related ingredient claim. This decision was based on the judgment that there is an implication that the food has particular nutritional properties when the ingredient is quantified.

Although energy and some antioxidants are not generally considered nutrients, claims related to energy and antioxidants were classified as nutrient content claims. A nutrient comparative claim is defined as 'a nutrition claim that compares the nutrient levels and/or energy value of two or more foods'. Therefore, a claim that a product was 'lite' was considered a nutrient comparative claim, as it implies that the product is lower in fat than a standard product. According to the INFORMAS taxonomy ${ }^{(7)}$, lite refers to fat, whereas 'light' could refer to colour or flavour, to which we applied the rule "if "light" is paired with a description of texture, taste or quality, for example, "light and crispy" it was not considered a claim, otherwise it was considered a nutrient comparative claim indicating "reduced fat"'. Similarly, 'baked not fried' was considered a nutrient comparative claim indicating that the product contains less fat than a similar product, which is fried. The phrase 'sweetened with stevia plant' was classified as a nutrient comparative claim, as it was believed that such a claim implied reduced sugar, as stevia is a known sugar substitute. For claim content, certain ingredients and nutrients were merged together for analysis purposes to avoid having too many categories; for example, antioxidants/vitamins/minerals/ hormones were combined and fruits/nuts/honey were combined.

In the case of claims that could be categorised as more than one type of claim, a hierarchy was applied according to the persuasive nature of the different claim types (online
Supplementary Appendix S1). For example, '100\% high oleic peanuts' implies the healthiness of peanuts (health-related ingredient claim) and states the presence of the nutrient high oleic acid (nutrient content claim). The hierarchy states that nutrient content claims take precedence over health-related ingredient claims, and thus this was classified as a nutrient content claim.

\section{Classification of products as 'healthy'/'less healthy'}

The FSANZ Health Claims NPSC 2014 was used to classify products as 'healthy' - that is, eligible to carry a health claim or 'less healthy' - that is, not eligible to carry a health claim ${ }^{(10)}$. The NPSC provides assessment of the overall nutritional composition of a food or beverage product by first applying 'baseline points' for energy, saturated fat, total sugar and $\mathrm{Na}$ content per $100 \mathrm{~g}$ and then applying 'modifying' points for dietary fibre ( $\mathrm{F}$ points), protein ( $\mathrm{P}$ points) and percentage of fruit and vegetables (including nuts and legumes, coconut, spices, herbs, fungi, seeds and algae) content ( $\mathrm{V}$ points). A final nutrient profiling score is given by subtracting the modifying points ( $\mathrm{V}, \mathrm{P}$ and $\mathrm{F}$ points) from the baseline points. $\mathrm{V}$ and $\mathrm{F}$ points were calculated based on the ingredient list and NIP, respectively, for the product (using photographs). In the case in which a $\mathrm{V}$ or $\mathrm{F}$ point could not be calculated for the product (percentage of fruit and/or vegetables or fibre content not mentioned in the ingredient list or NIP), a standard V point was used based on the most common percentage of fruit or vegetables for other products in the same category ${ }^{(10)}$.

In the case in which fibre content of the food was not known, a standard $\mathrm{F}$ point was used based on the average of fibre content for other products in the same food category.

For cheese products missing $\mathrm{Ca}$ content values, a standard value was used based on the Ca content value for similar cheeses provided in the Concise New Zealand Food Composition Tables, as insufficient values were present to calculate an average $\mathrm{Ca}$ content value ${ }^{(18)}$. Food products were classified as 'healthy' if the NPSC was $<4$, except for non-alcoholic beverages ('healthy' is NPSC <1) and cheese products containing $>320 \mathrm{mg} / 100 \mathrm{~g}$ of $\mathrm{Ca}$ ('healthy' is NPSC $<28)^{(10)}$. 


\section{Inter-rater reliability}

To evaluate the objectivity of the taxonomy for classification of claims, two researchers (H. H. A.-A. and A. D.) independently classified the claims in two food categories (snack foods ( $n$ 373) and biscuits $(n 728)$ ), and the percentage of different types of nutrition and health claims was compared. Discrepancies found were then solved between the researchers and a third researcher (S. V.).

\section{Statistical analyses}

Products with incomplete nutrition data, multiple NIP (such as variety packs) or duplicates in the database were excluded from the analysis ( $n$ 450). Microsoft Excel 2010 was used to determine the following: total number of claims in each food category, claim type and format type; the number of products carrying claims for each claim type and format type; and the number of 'healthy' and 'less-healthy' products for each claim type and for each claim content.

The NPSC score for each product was calculated using IBM SPSS Statistics 2.0. $\chi^{2}$ tests were performed to compare the number of different types of claims between two coders for two food groups. A $P$ value of 0.05 was considered statistically significant.

\section{Results}

A total of 7526 products representing eight food categories had complete data and were included in the analysis.

\section{Eligibility of food and beverage products to carry health claims}

Overall, 3557 (47\%) food products across eight categories were classified as 'healthy' according to the NPSC. Four of the eight food categories included a greater proportion of 'healthy' than 'less-healthy' products - that is: fruit/vegetables, convenience foods, dairy products and cereals. The snack foods category had the greatest proportion ( $n$ 338; 93\%) of products classified as 'less healthy' (Fig. 1; online Supplementary Appendix S2).

\section{Inter-rater reliability of classifying claims on food packages}

The percentage of biscuits and snack foods with claims and the percentage of different types of claims on the FoP of both snack foods and biscuits were similar for both independent researchers. There was only a significant difference for the percentage of health-related ingredient claims found on biscuits $(P=0 \cdot 021)$ (Table 2).

\section{Extent of nutrition and health claims on 'healthy' $v$. 'less-healthy' products}

Overall, more than one-third of all food products ( $n$ 2644; $35 \%$ ) featured at least one nutrition claim, whereas 15\% ( $n$ 1094) featured at least one health claim. 'Healthy' products displayed a proportionately greater percentage of both nutrition and health claims compared with 'less-healthy' products. Almost half ( $n$ 1596; $45 \%$ ) of all 'healthy' products displayed nutrition

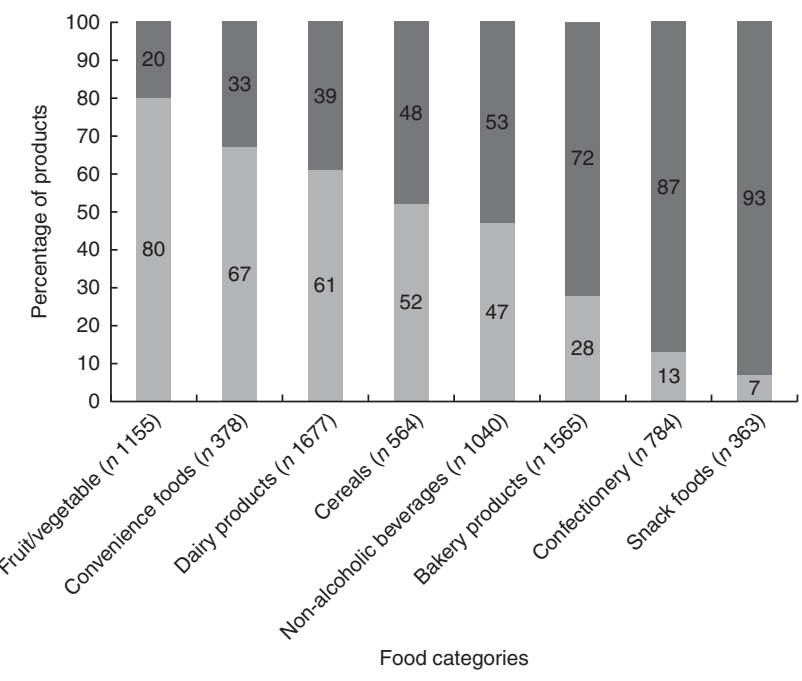

Fig. 1. Percentage of New Zealand packaged food products classified as 'healthy' $(\square)$ and 'less healthy' $(\square)$ by food category.

claims and almost one-quarter ( $n$ 807; 23\%) displayed health claims, whereas $26 \%$ ( $n$ 1048) of 'less-healthy' food products carried nutrition claims and $7 \%$ ( $n$ 287) carried health claims (online Supplementary Appendix S2). A total of 7058 individual claims were found, with nutrition claims ( $n$ 5454; 77\%) more prevalent than health claims ( $n$ 1604; $23 \%$ ) for both 'healthy' and 'less-healthy' products across all eight categories (Fig. 2). About $69 \%$ ( $n$ 4875) of nutrition and health claims were found on 'healthy' food products; these were predominantly nutrition claims ( $n$ 3650; 75\%). One-third of claims were found on 'less-healthy' products ( $n$ 2183), featuring a substantially greater proportion of nutrition claims (83\%) than health claims (17\%) (online Supplementary Appendix S3). Nutrition claims featured more frequently on 'less-healthy' products than health claims. This was the case for each of the eight food categories (Fig. 2).

The majority of health claims found on 'less-healthy' products were general health claims ( $n 353$; 93\%), of which most are not included as part of the FSANZ Health Claims legislation ${ }^{(10)}$. Furthermore, a number of reduction of disease risk claims were found on 'less-healthy' cereals ( $n$ 8), convenience foods ( $n$ 4) and dairy products ( $n$ 5) (online Supplementary Appendix S3). All seventeen of these were represented by the HFT, which is considered an endorsement and therefore is not subject to FSANZ Standard requirements ${ }^{(19)}$. Categories with the greatest proportion of 'less-healthy' foods (snack foods and confectionery; 93 and $87 \%$, respectively) featured a greater proportion of nutrition and health claims on 'less-healthy' foods than 'healthy' foods (online Supplementary Appendix S3).

The cereals category had a substantially greater proportion of products carrying claims than any other category ( $n 1503$ on 564 products), one-third of which were displayed on 'less-healthy' products ( $n$ 508) (online Supplementary Appendix S3).

\section{Nature of claims found on 'healthy' v. 'less-healthy' products}

Nutrient content claims were the most common type of nutrition claim on the FoP observed across most food categories (Fig. 3). 
Overall, nutrient content claims referring to fats were most prevalent. The snack foods category was an exception, as nutrient comparative claims were the most common nutrition claims (38\%) on these products, predominantly 'reduced fat' claims (online Supplementary Appendix S3). Almost all of the products carrying these nutrient comparative claims were 'less-healthy' products (fifty-five products) (online Supplementary Appendix S2). Of all 'reduced fat' claims, more than half were found on 'less-healthy' products ( $n 332 ; 52 \%$ ). Similarly, claims referring to energy (nutrient content and/or nutrient comparative claims) were predominantly found on 'less-healthy' products ( $n$ 227; 57\%) compared with 'healthy' products (data not shown).

Health claims were most commonly featured in the form of general health claims across all food categories ( $n$ 1197; 75\%) (Fig. 3). Convenience foods had the greatest proportion of health claims (33\%), with general health claims comprising $73 \%$. In confectionery and snack foods, general health claims were the sole type of health claims present (online Supplementary Appendix S3).

Reduction of disease risk claims represented $32 \%$ of health claims found on dairy products, which was the greatest proportion found in any category, with the HFT as the predominant content type ( $n$ 100; $92 \%$ ). The HFT represented $4 \%(n$ 297) of all claims, with 6\% ( $n$ 17) found on 'less-healthy' products (data not shown).

\section{Discussion}

This cross-sectional study provides an overview of the extent and nature of nutrition and health claims on the FoP of eight food categories of New Zealand packaged foods. Overall, more than half of all products across the eight categories featured claims on the FoP with a total of 7058 individual claims, indicating extensive use of nutrition and health claims on packaged foods in New Zealand. More than a quarter of 'less-healthy' products (26\%) featured nutrition claims and $7 \%$ carried health claims.

The proportion of cereals with claims (90\%) is markedly higher than for the other seven food categories. A previous New Zealand study on breakfast cereals using Nutritrack data from 2013 found that over a quarter of breakfast cereals were classified as 'less healthy' and $58 \%$ of 'cereals for kids' classified as 'less healthy'. This data are significant, as cereals constitute a regular part of children's and adult's diets, with cereals as common breakfast meals and cereal bars as common lunchbox items $^{(20)}$

It is noteworthy that a much higher proportion of nutrition than health claims were found in our study across all food categories. This might reflect the tight regulations that exist in New Zealand restricting the use of health claims on products classified as 'less healthy' according to the NPSC. Nutrition claims, however, are not restricted for use on 'less-healthy' foods. Therefore, claims such as 'low fat', 'sugar free' and 'baked not fried' can, and do, exist on 'less-healthy' products, potentially misleading consumers to perceive those products as healthier than they are. It was especially apparent that confectionery and snack foods, the two categories with 


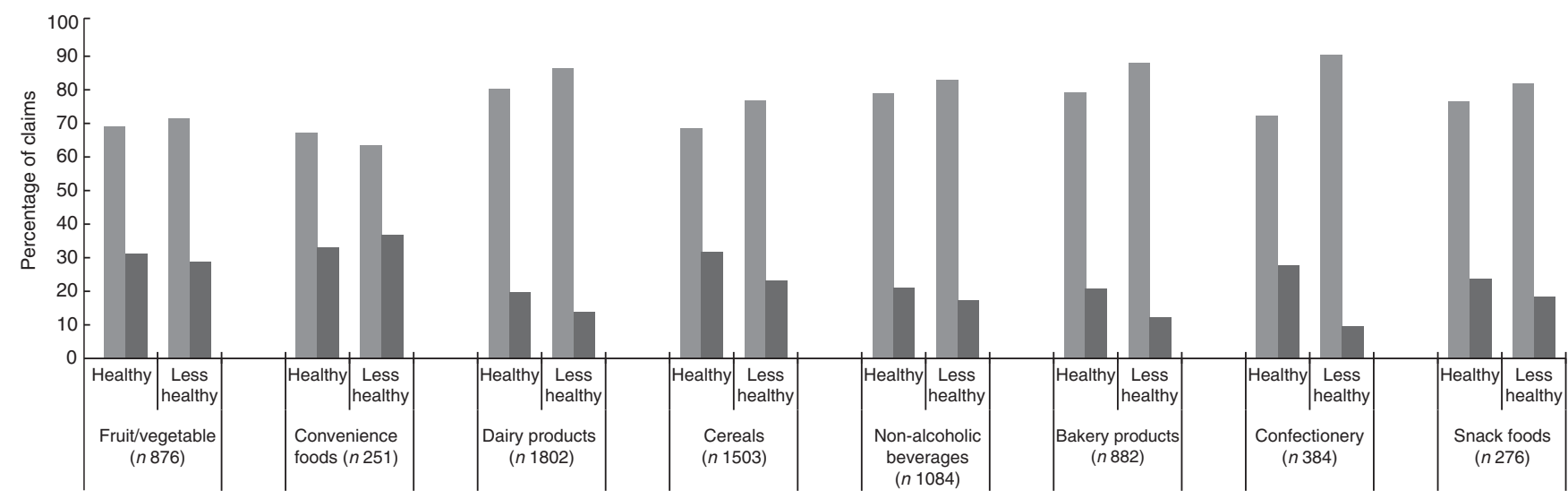

Fig. 2. Percentage of nutrition ( $\square$ ) and health claims $(\square)$ on 'healthy' and 'less-healthy' New Zealand packaged food products in each food category. $n$, total number of claims.

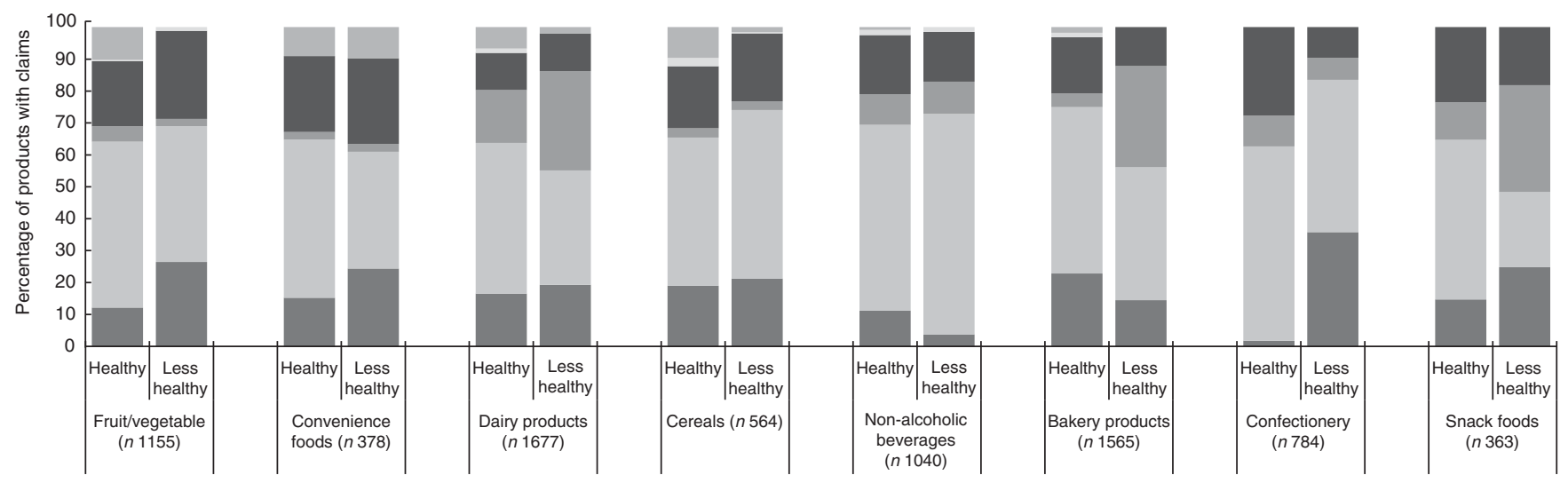

Fig. 3. Percentage of 'healthy' and 'less-healthy' New Zealand packaged food products with different types of nutrition and health claims in each food category. $n$, total number of products; , health-related ingredient claims; , nutrient content claims; , nutrient comparative claims; $\square$, general health claims; $\square$, nutrient and other function claims; , reduction of disease risk claims.

highest proportion of 'less-healthy' foods, carried many 'low fat', 'reduced fat' and 'reduced sugar' claims but no reduction of disease risk claims or nutrient and other function claims.

In all, seventeen reduction of disease risk claims as per the INFORMAS taxonomy were found on 'less-healthy' cereals in the form of the HFT, similar to the findings on New Zealand breakfast cereals ${ }^{(20)}$. However, according to the FSC the HFT is currently not considered a health claim and thus not subject to the legislation. In addition, general health claims were quite prevalent, such as 'good for you'. Most of such claims are not (yet) policy regulated, yet they may have an influential, and potentially misleading, role in consumer food choices.

A recent UK study, using the INFORMAS taxonomy, found similar results to this study. Notably, nutrition claims were significantly more common than health claims, and nutrient content claims were the most frequent type of claim, with fat identified as the most prevalent nutrient/ingredient mentioned and dairy products and non-alcoholic beverages carrying the most nutrition and health claims ${ }^{(21)}$. Our findings were also consistent with a similar US study ${ }^{(22)}$.
Previous research has reflected the misleading nature of nutrition claims. FSANZ assessed participants' understanding of nutrient claims and found that $77 \%$ thought that 'lite' referred only to fat, $28 \%$ thought that 'no added sugar' meant that the product was sugar-free, only $11 \%$ correctly interpreted 'reduced salt' and only $16 \%$ correctly interpreted the ' $94 \%$ fat-free' claim as a medium-fat food ${ }^{(23)}$. Furthermore, it has been found that labelling snacks as 'low fat' increases food intake during a single consumption occasion by up to $50 \%{ }^{(24)}$. Thus, the consumer's misguided perception reduces guilt associated with eating 'low-fat' food, which translates into greater consumption, potentially leading to weight gain ${ }^{(25)}$.

The overall good inter-rater reliability of classifying claims according to the international INFORMAS taxonomy reflects the consistency of coding the data using the definitions, rules and hierarchies we included in the taxonomy guidelines. This will aid consistency in repeat studies using the taxonomy in the future.

Although the focus of this study was on labels on the FoP, a weakness was that many products featured claims on all sides of their packaging. This was particularly difficult with canned products, as the 'sides' contained several health and 
nutrition claims but the area defined as FoP only had the brand name and product flavour. Therefore, although the FoP is the part that attracts the most attention by consumers, it underestimated the total number of claims visible to consumers, and future studies might need to include the full packages of the products.

This study highlights the extensive use of nutrition claims on packaged foods in New Zealand supermarkets, particularly on 'less-healthy' foods. These results reflect consumers' exposure to potentially misleading nutrition claims, and this should thus be recognised as a significant target for policy intervention.

The newly implemented FSANZ Standard 1.2.7 only regulates nutrient content claims and health claims. Consumers are continuously at risk of being misguided by food labels, featuring non-regulated claims, resulting in misinformed, possibly lesshealthy food choices. This INFORMAS taxonomy, with its adapted definitions of nutrition and health claims, captured claims that are not currently included in the FSC regulations and which could still misleadingly affect consumers' perceptions of food healthiness.

It needs to be explored how current regulations on nutrition and health claims in New Zealand could be strengthened (e.g. using the NPSC for nutrition claims, including general health claims as per the INFORMAS taxonomy) to ensure that consumers are protected and not misled.

\section{Acknowledgements}

The authors acknowledge the Health Research Council of New Zealand for funding this study (grant no.: 3704724). S. V. is supported by a fellowship from the National Heart Foundation of New Zealand. The funders had no role in the design, analysis or writing of the article.

S. V. and B. S. designed the study; H. H. A.-A. and A. D. collected and analysed the data; and H. H. A.-A. and S. V. prepared a draft version of the manuscript. All authors critically revised the manuscript.

The authors declare that there are no conflicts of interest.

\section{Supplementary material}

For supplementary material/s referred to in this article, please visit http://dx.doi.org/doi:10.1017/S0007114516002981

\section{References}

1. World Health Organization (2013) Global Action Plan for the Prevention and Control of Non-Communicable Diseases 2013-2020. Geneva: WHO.

2. World Health Organization (2014) Global Status Report on Noncommunicable Diseases 2014. Geneva: WHO.

3. Institute for Health Metrics and Evaluation (2013) Global burden of disease compare. http://vizhub.healthdata.org/ gbd-compare (accessed October 2015).

4. Ministry of Health (2015) Annual Update of Key Results 2014/ 15: New Zealand Health Survey. Wellington: Ministry of Health.
5. Swinburn BA, Sacks G, Hall KD, et al. (2011) The global obesity pandemic: shaped by global drivers and local environments. Lancet 378, 804-814.

6. Swinburn B, Sacks G, Vandevijvere S, et al. (2013) INFORMAS (International Network for Food and Obesity/NonCommunicable Diseases Research, Monitoring and Action Support): overview and key principles. Obes Rev 14, Suppl. 1, $1-12$.

7. Rayner M, Wood A, Lawrence M, et al. (2013) Monitoring the health-related labelling of foods and non-alcoholic beverages in retail settings. Obes Rev 14, Suppl. 1, 70-81.

8. Codex Alimentarius Commission (2004) Guidelines For Use of Nutrition and Health Claims, CAC/GL 23-1997. Rome: Food and Agriculture Organization of the United Nations/World Health Organization.

9. Food Standards Australia New Zealand (2013a) Nutrition Content Claims and Health Claims. Canberra: Food Standards Australia New Zealand.

10. Food Standards Australia New Zealand (2013b) Short Guide for Industry to the Nutrient Profiling Scoring Criterion (NPSC) in Standard 1.2.7. Nutrition, Health and Related Claims. Canberra: Food Standards Australia New Zealand.

11. Ministry for Primary Industries (2013) Nutrition Information Panel (NIP) - Standard 1.2.8. Wellington: Ministry for Primary Industries New Zealand.

12. Signal L, Lanumata T, Robinson JA, et al. (2008) Perceptions of New Zealand nutrition labels by Maori, Pacific and low-income shoppers. Public Health Nutr 11, 706-713.

13. Ni Mhurchu C, Brown R, Jiang Y, et al. (2015) Nutrient profile of 23596 packaged supermarket foods and non-alcoholic beverages in Australia and New Zealand. Public Health Nutr 19, 401-408.

14. Eyles H, Gorton D \& Ni Mhurchu C (2010) Classification of 'healthier' and 'less healthy' supermarket foods by two Australasian nutrient profiling models. $N Z$ Med $J \mathbf{1 2 3}$, 8-20.

15. New Zealand Food \& Grocery Council (2013) Daily intake labelling scheme. http://www.fgc.org.nz/education/dailyintake-labelling-scheme (accessed December 2015).

16. World Health Organization (2004) Nutrition Labels and Health Claims: The Global Regulatory Environment. Geneva: WHO.

17. National Institute for Health Innovation (2011) Nutritrack: reformulation of processed foods to promote health. http:// nihi.auckland.ac.nz/page/current-research/our-nutrition-andphysical-activity-research/nutritrack-reformulation-processe (accessed February 2015).

18. New Zealand Food Composition Database (2014) Concise New Zealand Food Composition Tables, 11th ed. Wellington: New Zealand Institute for Plant \& Food Limited/Ministry of Health.

19. Food Standards Australia New Zealand (2008) Nutrition, health and related claims - a short guide to the new standard. http://www.foodstandards.govt.nz/code/proposals/documents/ P293\%20FAR\%20Short\%20Guide.pdf (accessed April 2016).

20. Devi A, Eyles H, Rayner M, et al. (2014) Nutritional quality, labelling and promotion of breakfast cereals on the New Zealand market. Appetite 81, 253-260.

21. Kaur A, Scarborough P, Matthews A, et al. (2015) How many foods in the UK carry health and nutrition claims, and are they healthier than those that do not? Public Health Nutr 19, 988-997.

22. Brecher SJ, Bender MM, Wilkening VL, et al. (2000) Status of nutrition labeling, health claims, and nutrient content claims for processed foods: 1997 food label and package survey. J Am Diet Assoc 100, 1057-1062. 
23. Food Standards Australia New Zealand (2003) Food Labelling Issues: Quantitative Research with Consumers. Canberra: FSANZ.

24. Wansink B \& Chandon P (2006) Can "low-fat" nutrition labels lead to obesity? J Mark Res 43, 605-617.
25. La Fontaine HA, Crowe TC, Swinburn BA, et al. (2004) Two important exceptions to the relationship between energy density and fat content: foods with reduced-fat claims and high-fat vegetable-based dishes. Public Health Nutr 7, $563-568$. 